\title{
Einfluss einer dynamischen Auftragssteuerung nach dem Floating-Batch-Prinzip in zweistufigen Kommissioniersystemen mit Sortereinsatz
}

\author{
DiPL.-ING. MiCHAEL OTT, \\ PROF. DR. MiCHAEL TEN HOMPEL \\ LEHRSTUHL FÜR FÖRDER- UND LAGERWESEN, UNIVERSITÄT DORTMUND
}

\section{Zusammenfassung}

Im Rahmen eines Projektes der Deutschen Forschungsgesellschaft (DFG) wurden am Lehrstuhl für Förder- und Lagerwesen der Universität Dortmund die Auswirkungen einer dynamischen Auftragssteuerung nach dem Floating-Batch-Prinzip auf die Effizienz und das Systemverhalten zweistufiger Kommissioniersysteme untersucht. Hierbei wurden Potenziale, technische und organisatorische Voraussetzungen sowie Grenzen für dessen Einsetzbarkeit abgeleitet, sowie geeignete Lösungen für bei der Umsetzung auftretende Problemstellungen ermittelt. Grundlage dieser Untersuchungen bildet eine detaillierte Analyse realisierter zweistufiger Kommissioniersysteme.

\begin{abstract}
In the frame of a project by the Deutsche Forschungsgesellschaft (DFG) the department of Transportation and Warehousing at the University of Dortmund studied the effects of a dynamical order managemend according to the floating batch principle onto the efficiency and system behaviour of two-level orderpicking systems. In this project the potentials, technical and organisational requirements as well as their application limits were determined and suitable solutions were developed for problems which may occur during the implementation. These studies were based on a detailed analysis of implemented two-level order-picking systems.
\end{abstract}

\section{Einleitung}

Kommissioniersysteme mit hohen Leistungsanforderungen werden bei mittleren bis großen Artikelspektren häufig zweistufig ausgeführt. Kennzeichen der zweistufigen Kommissionierung ist eine artikelorientierte Arbeitsweise, bei der die Prozesse Entnahme und Zuordnung zu einem Kundenauftrag voneinander getrennt sind. Die eingehenden Kundenaufträge werden anhand ausgewählter Kriterien in Auftragsgruppen, so genannten Batches, zusammengefasst und gemeinsam bearbeitet. Bei der Entnahme der Artikel besteht somit kein Bezug mehr zum einzelnen Kundenauftrag. Durch diese Verdichtung müssen die Entnahmeeinheiten zwangsläufig in einer zweiten Stufe, dem Sortier- und Verteilsystem, auf die externen Kundenaufträge verteilt werden (Abb. 1). 


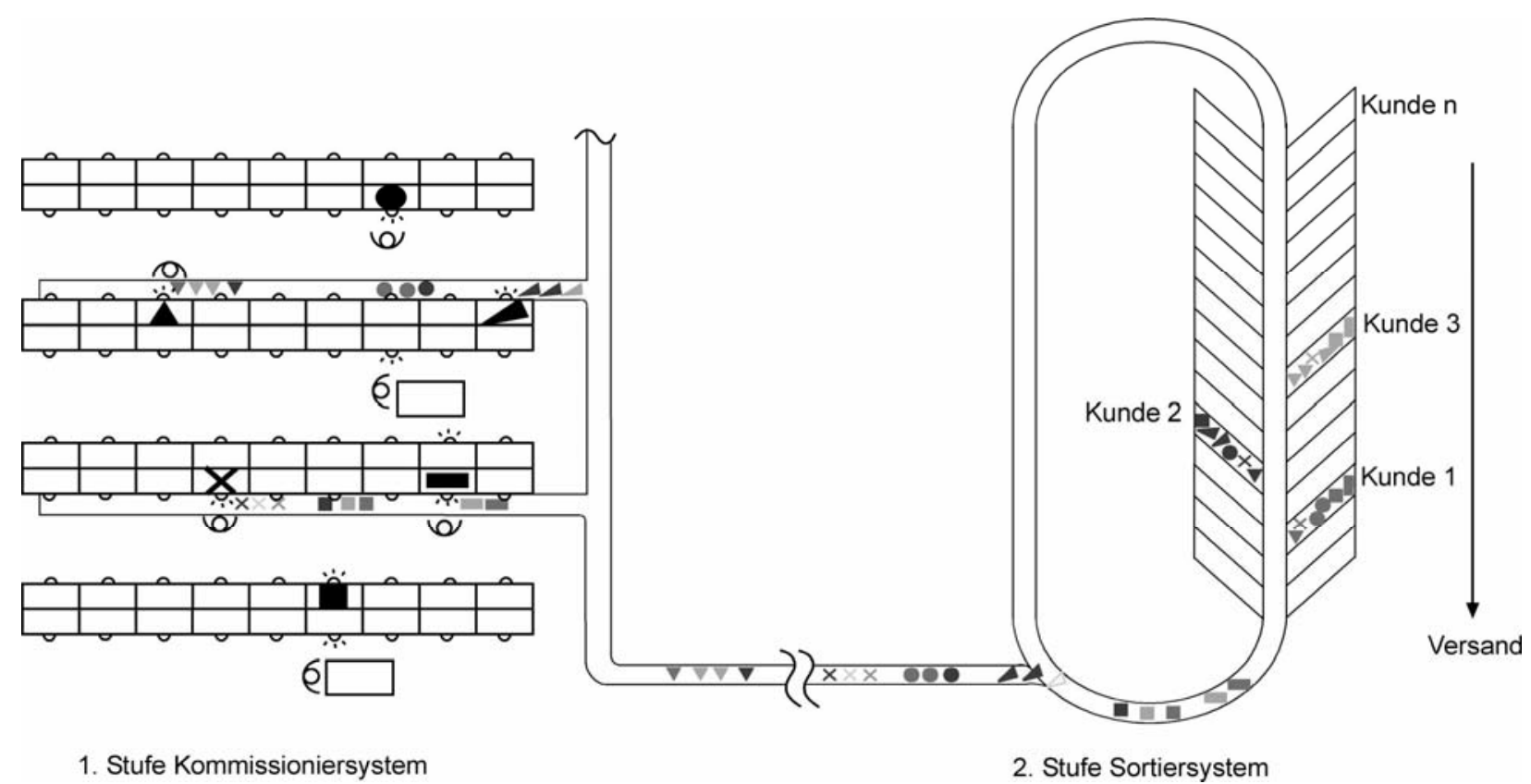

Abbildung 1: Zweistufige Kommissionierung mit Sortereinsatz

Der Batchbetrieb stellt eine Abwägung zwischen Vorteilen in der Kommissionierung und Nachteilen durch die Sortierung dar. Während in der ersten Stufe Einsparungen durch Zusammenfassung mehrerer Kundenauftragspositionen zu einer Kommissionierauftragsposition erzielt werden können, sind eine komplexere Steuerung der Ablauforganisation (z.B. Auftragsaufbereitung) sowie zusätzliche Transport-, Handlings- und Identifizierungsvorgänge in der Sortierung erforderlich. Ziel bei der Ausgestaltung eines zweistufigen Kommissioniersystems muss es daher sein, dass die in der ersten Kommissionierstufe erzielten Rationalisierungsgewinne größer sind, als die mit der zweiten Stufe verbundenen zusätzlichen Aufwände.

\section{Neue Herausforderungen}

Bedingt durch abnehmende Sendungsgrößen, kürzere Reaktionszeiten bei gleichzeitig hoher Lieferqualität, sowie die insgesamt zunehmende Anzahl an Warensendungen, haben die Anforderungen an Leistungsfähigkeit und Flexibilität zweistufiger Kommissioniersysteme deutlich zugenommen. Als Folge werden immer leistungsfähigere und damit auch kostenintensivere Kommissioniersysteme realisiert. Bei der Bereitstellung der Informationen für die Kommissionierer ermöglichen Übertragungstechniken wie beispielsweise Pick-by-Light oder Pick-by-Voice hohe Pickleistungen. Moderne Sortier- und Verteiltechniken zeichnen sich durch immer höhere Sortierleistungen aus. Im Rahmen von theoretischen Analysen zeigt sich jedoch, dass die Leistung eines mehr-stufigen Kommissioniersystems entscheidend vom Zusammenspiel der Teilsysteme abhängt. Dies lässt sich auch durch Beobachtungen in der Praxis verifizieren.

Einen wesentlichen Einfluss auf die Effizienz und Leistungsfähigkeit eines zweistufigen Kommissioniersystems haben die eingesetzte Strategie zur Auftragssteuerung (Abb. 2) und die entsprechenden Strategieparameter, wie beispielsweise Batchgröße und Freigabekriterien. Während herkömmliche statische Batchstrategien zu starken Leistungsschwankungen und Leistungsverlusten durch Wartezeiten führen, lässt sich die Produktivität eines Kommissioniersystems durch eine dynamische Batchstrategie erhöhen. 
Festes Batch

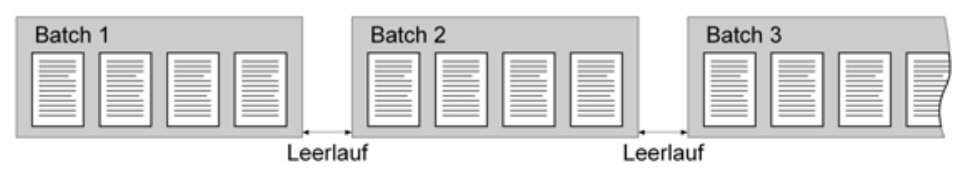

Festes überlappendes Batch

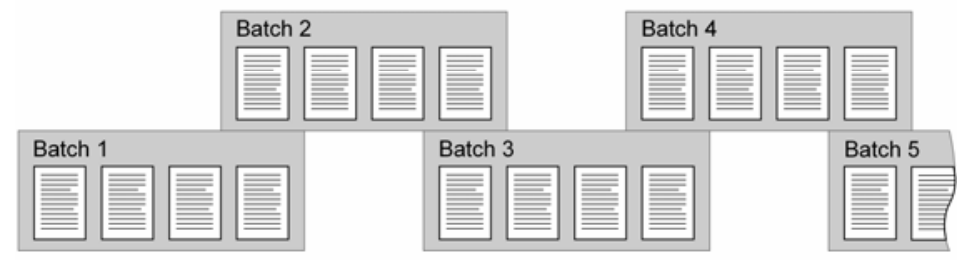

Dynamisches Batch

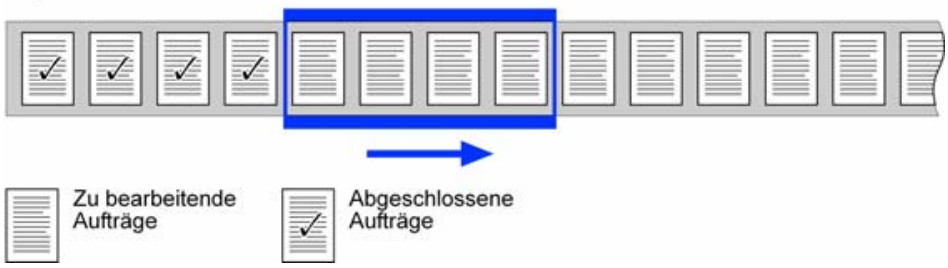

Abbildung 2: Strategien zur Auftragssteuerung

\section{Stand der Technik}

Bis heute gelangt im Bereich der zweistufigen Kommissionierung in vielen Fällen eine statische Auftragssteuerung nach dem so genannten Fixed-Batch-Verfahren (FBoÜ) zum Einsatz. Dieses sieht im Wesentlichen eine zeitliche Trennung des Pickprozesses und des Verteilprozesses vor. Da die Bearbeitung eines neuen Batches in der Kommissionierung erst beginnt, wenn alle Teile des vorhergehenden Batches verteilt und die Endstellen durch die Packer geleert sind, entstehen Wartezeiten bei den Kommissionierern. Durch die vollständige Trennung der beiden Kommissionierstufen ohne direkte Rückkopplung ergeben sich zwischen zwei Batches auch auf dem Sortierkreislauf zwangsläufig Leerlaufzeiten. Diese so genannten Batchwechselzeiten sind weitgehend konstant und beinhalten u.a. die Zeiten zum Füllen des Sortierkreislaufs über die Einschleusung, zur Überbrückung der Entfernung zwischen Aufgabe- und Endstellen und dem Entleeren des Sorters über das Packsystem. Ebenfalls berücksichtigt werden Zeiten zur Bearbeitung von No-Needs (Überhänge infolge Fehlkommissionierung), No-Reads (Fehllesungen) und Fehlteilen durch Pickfehler oder mangelnde Verfügbarkeit der Artikel.

Wenn durch Pick- oder Lesefehler Teile in einer Endstelle fehlen, so erfolgen keine Fertigmeldung der Aufträge und somit auch keine Freigabe der entsprechenden Endstelle. Es wird eine manuelle Nachbearbeitung erforderlich, die wiederum zu einem weiteren Anstieg der Batchwechselzeiten führt.

Verstärkt wird dieser Effekt durch die Charakteristik eines Batchlaufes, die in Abbildung 3 dargestellt ist. Nach dem Batchstart (BS) steigen die Anzahl der aktiven Aufträge, und damit die Anzahl der aktiven Endstellen, erst langsam an. Ebenso läuft sie am Batchende (BE) fließend aus. Dies führt zwangsläufig zu einer Unterauslastung des Sortier- und des Packsystems und somit zu einer Verringerung der Auslastung der Ressourcen. 

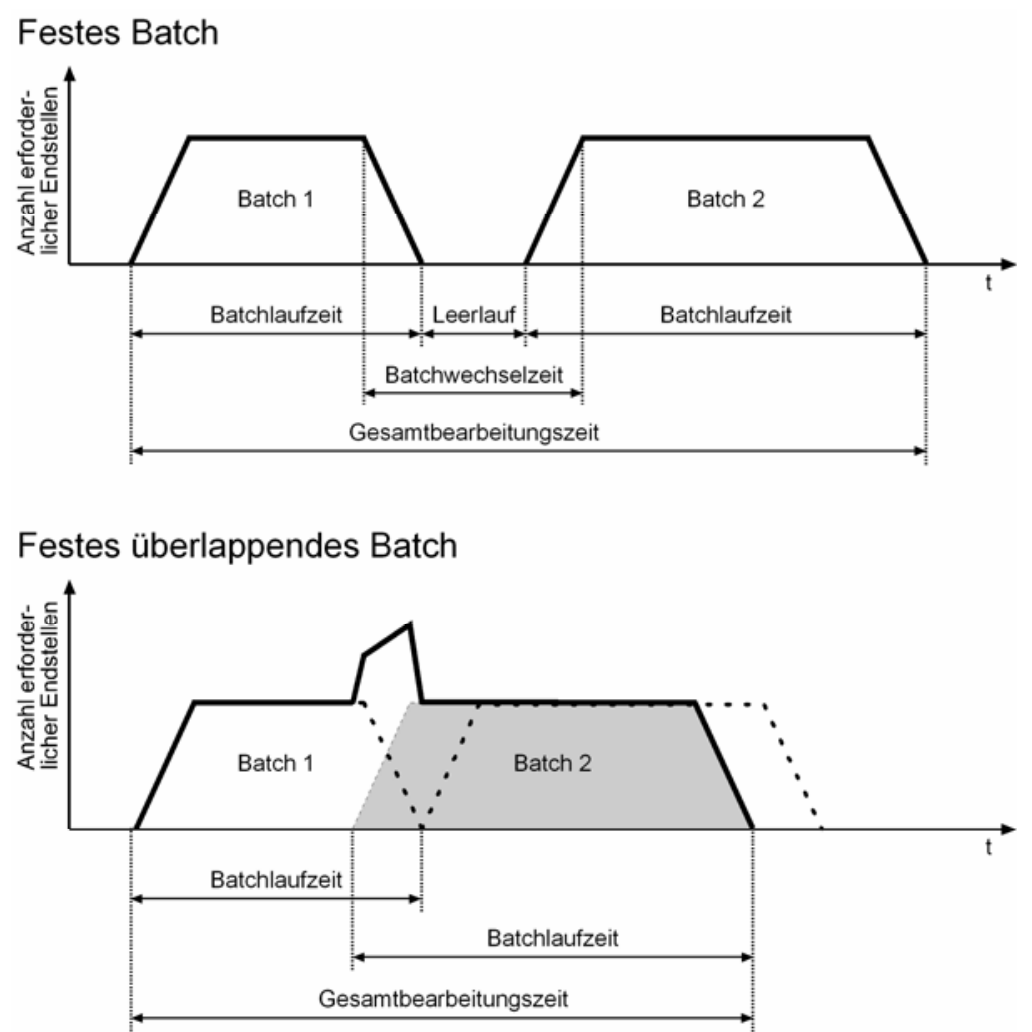

Abbildung 3: Anzahl erforderlicher Endstellen über dem Batchverlauf

Ein Ansatz zur Verbesserung der produktiven Sorterlaufzeit besteht in der Möglichkeit, die einzelnen Batches fester Größe früher freizugeben. Der Vorteil dieser Vorgehensweise, die als Überlappendes- oder OverlappingBatch-Verfahren (FBmÜ) bekannt ist, liegt im höheren erreichbaren Durchsatz durch eine Reduzierung der Batchwechselzeiten. Die Freigabe eines neuen Batches erfolgt jedoch frühestens, wenn das letzte Teil des aktuellen Batches das Kommissioniersystem verlassen hat. Durch diese Vorgehensweise kommt es zu einer Überlappung der Batches (Abb. 3), d.h. im Stückgutstrom des Sortiersystems befinden sich Teile von Batch i vor Teilen von Batch i-1. Während der Überlappungszeit entsteht ein erhöhter Bedarf an freien Endstellen, da bereits Aufträge des neuen Batches am Sorter eintreffen, während ein Teil der vorhandenen Endstellen noch durch Aufträge des vorangegangenen Batches belegt sind. Übersteigt die Anzahl der erforderlichen Endstellen in diesem Zeitraum die Anzahl der vorhandenen, so müssen Aufträge auf dem Sortierkreis rezirkulieren bis eine entsprechende Endstelle frei wird. In dieser Situation kann es im schlimmsten Fall zu einem Deadlock des Systems kommen, der ein manuelles Abräumen des Sorters notwendig machen kann. Dies muss in der praktischen Anwendung in jedem Fall vermieden werden. Mindestens wird jedoch die verfügbare Sorterkapazität durch die rezirkulierenden Teile eingeschränkt.

Sowohl beim festen, als auch beim überlappenden Batch bildet die Betriebsgrundlage eine Sequenz abgeschlossener Batches fester Größe, die schubweise in das System eingelastet wird. Das gesamte System arbeitet nach dem Push-Prinzip. Auf Grund dieser Arbeitsweise kommt es zu einer stoßweisen Belastung der Teilbereiche des Systems. Während diese auf die Maximallast ausgelegt werden müssen, fällt die Auslastung beider Teilsysteme während der Batchwechselzeiten stark ab. Zur Analyse unterschiedlicher Fragestellungen im Bereich fester Batches mit oder ohne Überlappung wurden in der Vergangenheit bereits umfangreiche Untersuchungen durchgeführt (u.a. [Bozer1983], [Bozer1988], [Radtke2000]). Eine Reihe wissenschaftlicher Beiträge konzentriert sich auf die Optimierung der Systemleistung durch die Variation der Batchgröße, die Ermittlung von Startzeitpunkten, die Zuweisungsstrategien oder die Berechnung der Anzahl und Kapazitäten der Endstellen ([Bozer1985], [Meller1997] und [Johnson1998]) sowie auf mögliche Leistungssteigerungen durch überlappende feste Batches ([Bozer1988], [Radtke2000]).

Bei den genannten Untersuchungen bildet die Betriebsgrundlage jedoch in allen Fällen eine Sequenz abgeschlossener Batches, die schubweise in das System eingelastet werden. 


\section{Dynamische Auftragssteuerung}

Einen Ansatz zur Harmonisierung der Systemauslastung und zur Reduktion der unwirtschaftlichen Leerlaufzeiten liefert das Prinzip der dynamischen Batchsteuerung, das so genannte Dynamische- oder FloatingBatch-Prinzip (DB). Herkömmliche statische Batchstrategien führen auf Grund der beschriebenen Zusammenhänge zu starken Leistungsschwankungen und Leistungsverlusten durch Wartezeiten. Aufgrund der dynamischen Arbeitsweise lässt sich beim Floating-Batch-Prinzip darauf schließen, dass eine Verbesserung der Produktivität zweistufiger Kommissioniersysteme erzielt werde kann.

Bisher wurde die Untersuchung der Auswirkungen einer dynamischen Auftragssteuerung nach dem FloatingBatch-Prinzip in zweistufigen Kommissioniersystemen in der Literatur noch nicht behandelt. Wissenschaftliche Untersuchungen, die allgemeinverbindliche und abgesicherte Aussagen zu Potenzial und Grenzen dynamischer Auftragssteuerungssysteme liefern, fehlen bislang völlig. In Fachbüchern wird das Floating-Batch-Prinzip vereinzelt als theoretische Möglichkeit zur Auftragssteuerung genannt ([Jünemann1999], [Gudehus2005]). Hierbei erfolgt jedoch keine eingehende Beschreibung und auch dort wird festgestellt, dass bisher keine Untersuchungen über die Einsatztauglichkeit vorliegen [Jünemann1999]. In Fachzeitschriften für Systemanwender oder in Tagungsbeiträgen wird vereinzelt von Floating-Batch-Realisierungen berichtet. Im Fokus dieser Veröffentlichungen stehen werbewirksame Aussagen in Form von Projektierungsberichten, die als Grundlage für eine vergleichende Untersuchung nicht geeignet sind.

Vor diesem Hintergrund hat sich der Lehrstuhl für Förder- und Lagerwesen im Rahmen eines Projektes der Deutschen Forschungsgesellschaft zum Ziel gesetzt, für zweistufige Kommissioniersysteme die Auswirkungen auf das Systemverhalten, sowie mögliche Effizienzsteigerungen durch den Einsatz des Floating-Batch-Prinzips zu untersuchen.

\subsection{Arbeitsweise und Zielsetzung}

Das Floating-Batch-Prinzip grenzt sich gegenüber den beiden vorgenannten Strategien zur Auftragssteuerung durch drei wesentliche Aspekte ab. Dies sind:

- $\quad$ die Umkehrung vom Push- zum Pull-Prinzip,

- $\quad$ die Auflösung der statischen Batches und

- $\quad$ die Vorgehensweise bei der Festlegung der Auftragsreihenfolge.

Anders als bei den klassischen Batchstrategien wird nicht ein Batch fester Größe nach dem anderen in das System eingelastet. Stattdessen werden einzelne Kundenaufträge aus dem Auftragsstapel entfernt, sobald diese abgeschlossen sind und damit eine Endstelle für einen neuen Auftrag zur Verfügung steht. Jeder aus dem Auftragsstapel entfernte Auftrag zieht nach dem Pull-Prinzip einen neuen Auftrag aus dem Auftragspool in das System (Abb. 2). Im Gegensatz zu den anderen Strategien handelt es sich somit bei der dynamischen Auftragssteuerung nicht um einen festen statischen Auftragsstapel, vielmehr findet eine kontinuierliche Veränderung des Auftragsstapels statt.

Für den Auftragsstapel wird in der Systemsteuerung eine bestimmte Anzahl gleichzeitig aktiver Aufträge festgelegt, die mit der Batchgröße bei statischen Batches verglichen werden kann und einen wesentlichen Strategieparameter darstellt.

Durch das Einspielen neuer Aufträge in den aktuellen Auftragsstapel ändert sich bei der dynamischen Auftragssteuerung die Reihenfolge der durch den Kommissionierer zu bearbeitenden Positionen. Die Vorgabe der zu kommissionierenden Positionen erfolgt üblicherweise wegoptimiert. Liegen die Entnahmeorte neu eingespielter Positionen in Laufrichtung vor den Entnahmeorten der bereits im Auftragsstapel enthaltenen Auftragspositionen, so ist eine Aktualisierung der Informationen zur Kommissioniererführung erforderlich. Die neuen Positionen werden zuerst kommissioniert, so dass sich die Bearbeitung der „älteren“ Positionen verzögert. Beim Floating-Batch-Prinzip besteht die Möglichkeit, die Festlegung der Auftragsreihenfolge und das Einspielen neuer Aufträge über einen dynamischen Zuweisungsprozess zu realisieren, bei dem die Auftragsreihenfolge kurzfristig verändert werden kann. Basierend auf aktuellen Informationen über den Status der Aufträge (Auftragsstati) sowie des Kommissionier- und des Sortiersystems (Systemstati) kann zustandsabhängig der am besten geeignete Folgeauftrag für die Bearbeitung ausgewählt werden.

Aus den beschriebenen Unterschieden lassen sich unmittelbar die Ziele ableiten, die mit dem Einsatz der dynamischen Auftragssteuerung verfolgt werden. 
Dies sind:

- das Erreichen einer höheren Systemleistung und eines höheren Durchsatzes,

- $\quad$ eine gleichmäßige Auslastung der Teilsysteme durch eine Harmonisierung der Leistungsanforderungen und

- $\quad$ eine höhere Flexibilität gegenüber kurzfristigen Ereignissen wie z.B. Fehlbeständen oder Eilaufträgen.

Die Gesamtleistung des Kommissioniersystems wird in starkem Maße von der Entnahmeleistung der ersten Stufe bestimmt. Zur Steigerung der Kommissionierleistung muss die für die Bearbeitung sämtlicher Auftragspositionen erforderliche Kommissionierzeit reduziert werden. Ausgehend von einem Kommissioniersystem mit Pick-to-Belt-Prinzip und einer Online-Übertragung der Entnahmeinformationen können die Basiszeiten unberücksichtigt bleiben. Da die Anzahl der insgesamt zu kommissionierenden Teile durch die dynamische Auftragssteuerung nicht beeinflusst wird, bleibt die erforderliche Greifzeit konstant. Beim Einsatz der dynamischen Auftragssteuerung wird die Kommissionierzeit somit durch zwei Effekte beeinflusst. Einerseits kann eine Reduzierung der bei einer statischen Auftragssteuerung auftretenden Wartezeiten (Totzeit) zwischen zwei Batches angenommen werden. Andererseits wirkt sich die dynamische Auftragssteuerung in Verbindung mit den aufgezeigten Einflussgrößen auf die Entnahmedichte und damit auf die zurückzulegenden Wege (Wegzeit) in der Kommissionierung aus. Tendenziell ergeben sich daher die in Abbildung 4 dargestellten Beeinflussungsmöglichkeiten.

Es ist somit zu untersuchen, unter welchen Bedingungen sich durch den Einsatz einer dynamischen Auftragssteuerung eine höhere Systemleistung erzielen lässt.

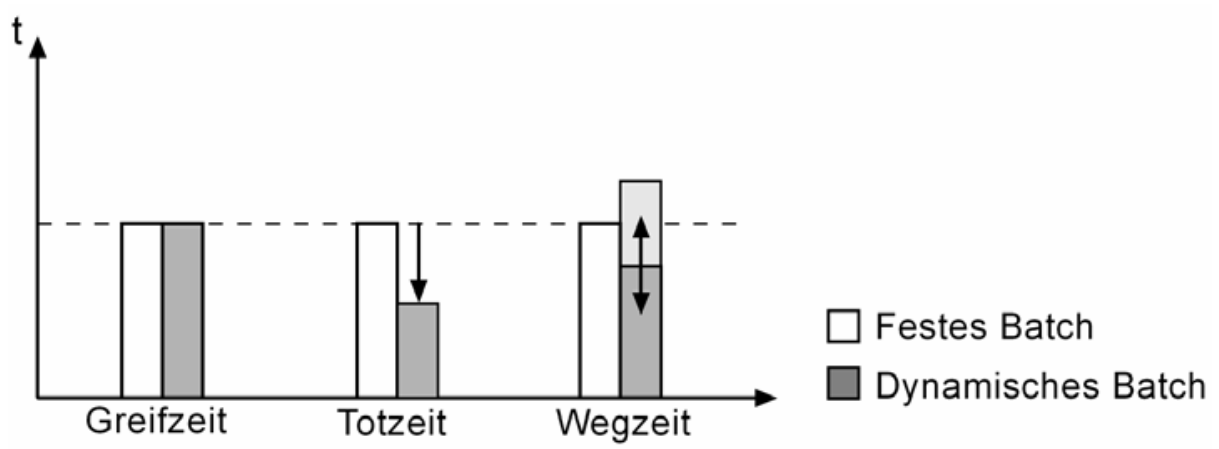

Abbildung 4: Mögliche Beeinflussung der Kommissionierzeitanteile durch dynamische Auftragssteuerung

Abbildung 5 zeigt qualitative Belastungsverläufe für die alternativen Strategien zur Auftragssteuerung. Beim Festen-Batch fällt die Belastung der Teilsysteme bis auf Null ab. Außerdem arbeiten die Teilsysteme im Bereich der An- und Auslaufphasen mit deutlicher Unterauslastung. Durch die Überlappung der Batches kann das Leerlaufen des Systems vermieden werden. Dennoch verbleiben Phasen in denen die Teilsysteme mit schlechter Auslastung arbeiten. Ziel der dynamischen Arbeitsweise des Floating-Batch-Prinzips ist es, durch die Vermeidung von Leerlauf- bzw. An- und Auslaufphasen zwischen zwei Batches, eine gleichmäßige und durchgehend hohe Leistung der Teilsysteme zu erreichen (Abb. 5) und die beim klassischen Batchbetrieb auftretenden Wartezeiten (Totzeiten) zu vermeiden.

Die Auswahl des Folgeauftrages erfolgt bei der dynamischen Auftragssteuerung erst unmittelbar vor dem Einspielen des Auftrages. Hierdurch bietet sich die Möglichkeit, die Auftragsreihenfolge im Auftragspool kurzfristig zu verändern und aktuelle Auftrags- und Systemstati zu berücksichtigen. Dies bedeutet ein Höchstmaß an Flexibilität gegenüber kurzfristigen Einflüssen wie z.B. Fehlbeständen, blockierten Endstellen oder Eilaufträgen. 

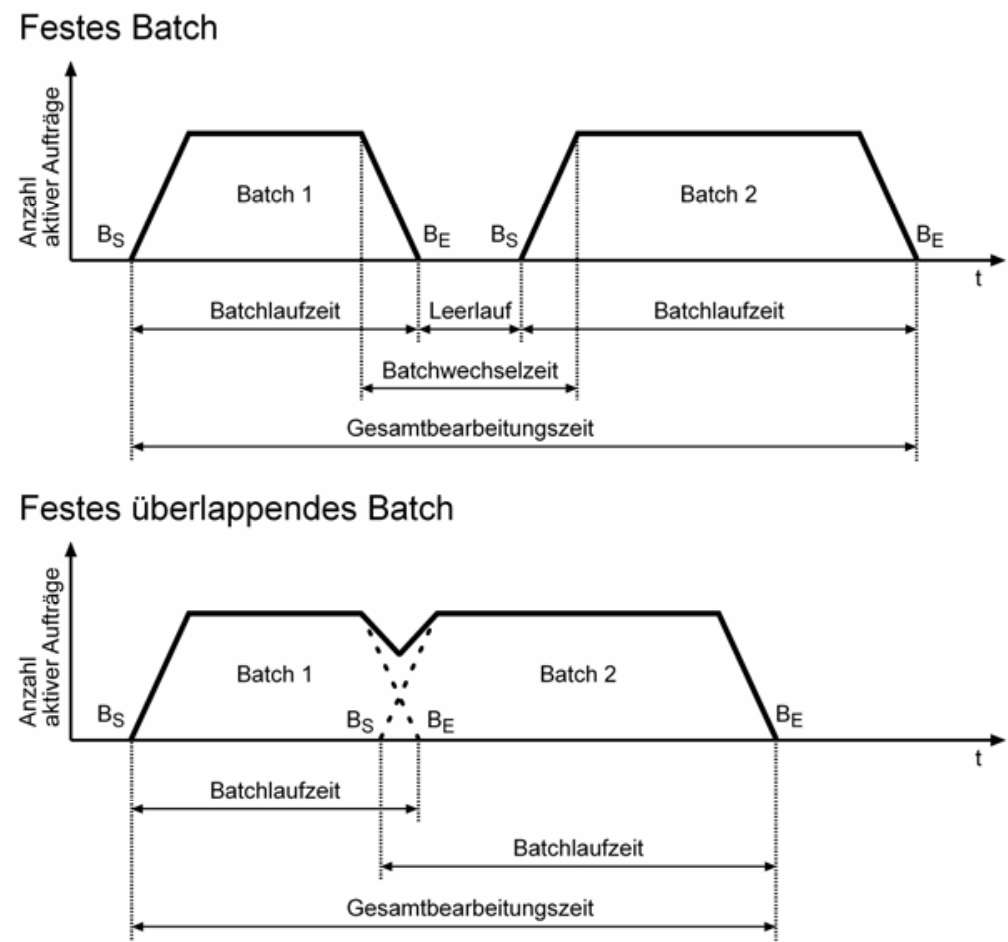

Dynamisches Batch

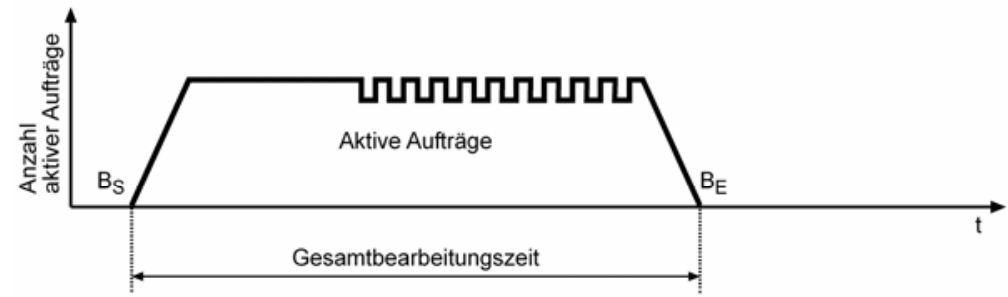

$\mathrm{B}_{\mathrm{S}}=$ Batchstart $\quad \mathrm{B}_{\mathrm{E}}=$ Batchende

Abbildung 5: Typische Belastungsverläufe der unterschiedlichen Strategien zur Auftragssteuerung

\subsection{Voraussetzungen und Einschränkungen}

Die zur Integration einer dynamischen Auftragssteuerung nach dem Floating-Batch-Prinzip erforderlichen Voraussetzungen sind die zeitnahe informationstechnische Kopplung der Teilsysteme mittels eines leistungsfähigen EDV-Systems und die direkte materialflusstechnische Kopplung der Teilsysteme ohne zeitliche Verzögerung. Das EDV-System muss die Verfügbarkeit stets aktueller Daten über den Status der einzelnen Kundenaufträge im Kommissioniersystem und die schnelle Reaktion auf diese Informationen gewährleisten. Hierfür müssen entsprechende Identifizierungspunkte vorhanden sein, die eine Rückmeldung über die Fertigstellung eines einzelnen Auftrages als Trigger für den Folgeauftrag ermöglichen. Die Übertragung der Entnahmeinformationen an die Kommissionierer muss mittels Online-Verfahren erfolgen, da sich der vom Kommissionierer zu bearbeitende Auftragsstapel während der Kommissionierung ständig ändert. Die Anzeige der Informationen erfolgt positionsweise, d.h. eine neue Auftragsposition wird angezeigt, sobald die vorangegangene Position durch den Kommissionierer quittiert wurde. Durch die Quittierung jeder Entnahme ist dem übergeordneten Leitsystem der genaue Bearbeitungsfortschritt der Kommissionierung bekannt und kann bei der Auswahl der nächsten Aufträge genutzt werden.

Insgesamt ergeben sich hierdurch höhere technische Anforderungen an das Steuerungssystem und die Kommunikationstechnik als bei einer statischen Auftragssteuerung. Die im Rahmen des Projektes durchgeführten Analysen realer zweistufiger Kommissioniersysteme haben jedoch gezeigt, dass die meisten modernen Kommissioniersysteme diese Anforderungen bereits erfüllen.

Die zweite wesentliche Voraussetzung ist die verzögerungsfreie Zuführung der entnommenen Teile zum Sortiersystem. Dies ist erforderlich, um die Reaktionszeit zwischen der vollständigen Entnahme eines Auftrags und dem Einspielen des Folgeauftrags in den Auftragsstapel gering zu halten. Eine hohe Reaktionszeit wirkt sich negativ auf die Entnahmedichte und somit letztlich auf die Kommissionierleistung bei dynamischer Auftragssteuerung aus. 
Prinzipiell stellt diese Anforderung gleichzeitig einen Vorteil der dynamischen Auftragssteuerung dar, da bei der Planung eines neuen Kommissioniersystems auf Zwischenpuffer zur Entkopplung der Systeme verzichtet werden kann. Durch die Einsparung der entsprechenden Technik werden die erforderlichen Investitionskosten reduziert.

Logische Einschränkungen für mögliche Einsatzbereiche einer dynamischen Auftragssteuerung nach dem Floating-Batch-Prinzip ergeben sich aus der Systemgröße und Systemkomplexität eines Kommissioniersystems. In großen Kommissioniersystemen ist häufig eine Konsolidierung der Batches in einem Puffer zur Entkopplung der Teilsysteme unumgänglich, um die stark schwankenden Bearbeitungszeiten in den unterschiedlichen Kommissionierzonen, sowie die hohen und sehr unterschiedlichen Transportzeiten zu synchronisieren. Dies widerspricht jedoch dem Grundgedanken der dynamischen Auftragssteuerung.

Darüber hinaus ist der Einsatz des Floating-Batch-Prinzips dann nicht sinnvoll, wenn eine gleichmäßige und durchgehend hohe Systemauslastung unerwünscht ist bzw. der hieraus entstehende Vorteil nicht genutzt werden kann. Als Beispiel sei das Zentrallager eines Unternehmens im Bereich des Lebensmittelhandels genannt. Die Kommissionierung erfolgt teilweise in gekühlten Kommissionierzonen. Für die hier tätigen Mitarbeiter sind in regelmäßigen Abständen kurze Aufwärmzeiten außerhalb des gekühlten Bereiches vorgeschrieben. Zur Einhaltung dieser Vorschrift werden die Laufzeit der Batches und die Leerlaufzeiten während des Batchwechsels hieran angepasst. Bei dynamischer Auftragssteuerung wären in diesem Fall Zwangspausen oder eine rotierende Arbeitsweise unter Einsatz zusätzlicher Mitarbeiter erforderlich. Für den Betrieb eines solchen Systems ist die dynamische Auftragssteuerung somit nicht sinnvoll.

\subsection{Störgrößen und Lösungen}

Bedingung für das Einspielen eines neuen Auftrages ist bei der dynamischen Auftragssteuerung, dass ein Auftrag des aktuellen Auftragsstapels abgeschlossen wird. Es muss daher sichergestellt werden, dass jeder eingespielte Auftrag in definierter Zeit abgeschlossen werden kann. Problematisch wird es, wenn durch Fehler im Bereich des Kommissioniersystems (z.B. falsche Entnahmemenge oder falscher Artikel kommissioniert) oder des Sortiersystems (z.B. Artikel nicht identifizierbar) ein Teil eines Auftrags nicht am Identifikationspunkt eintrifft. Liegt dem Steuerungssystem keine Information darüber vor, dass diese Artikeleinheit nicht mehr eintreffen wird, so kann der entsprechende Auftrag nicht abgeschlossen und die entsprechende Endstelle nicht freigegeben werden. In der Konsequenz kann somit im Kommissioniersystem kein neuer Auftrag eingespielt werden. Tritt dieser Fehler häufiger auf, so sinkt im Kommissioniersystem die Entnahmedichte immer weiter ab und im Sortiersystem bleiben immer mehr Endstellen belegt.

Wird der fehlende Artikel zu einem späteren Zeitpunkt in der Kommissionierung erneut angesprochen, so wird er gegebenenfalls beim Eintreffen am Sorter derjenigen Endstelle zugewiesen, in der er zuvor nicht angekommen ist (First-Come-First-Serve-Prinzip). Hierdurch kann der unvollständige Auftrag zwar abgeschlossen werden, jedoch verlagert sich das Problem auf einen anderen Auftrag und bleibt prinzipiell erhalten. Der Fehler wird solange weiter gereicht, bis keine Entnahmeeinheiten des Artikels mehr am Sorter eintreffen. Erst jetzt wird der Fehler zwangsläufig auffallen. Durch die entstehenden Verzögerungen steigt die Durchlaufzeit der betroffenen Aufträge und im Extremfall können sie nicht termingerecht fertig gestellt werden.

Die Lösung dieses Problems kann über einen Zeitschrankenmechanismus erfolgen, bei dem zunächst eine maximale Zeitspanne (Zeitschranke) definiert wird, innerhalb der alle Teile eines Auftrages in der Endstelle eintreffen müssen. Anschließend erfolgt eine Statuskontrolle anhand der Rückmeldungen aus dem Kommissionier- und Sortiersystem. Für jede Auftragsposition wird ein Datumsfeld mitgeführt, in dem die Zeit der letzten Änderung (gepickt, am Sorter identifiziert,...) als Zeitstempel abgespeichert wird. Über diesen können diejenigen Aufträge identifiziert werden, die schon lange im System sind und eine Endstelle belegen. Überschreitet der Zeitstempel die definierte Zeitschranke, so erfolgt eine Meldung an den zuständigen Packer, der das Problem beseitigen und den Auftrag manuell abschließen kann.

\subsection{Einflussgrößen und Zielfaktoren}

Grundsätzlich sind bei dynamischer Auftragssteuerung in einigen Bereichen ähnliche Effekte zu erwarten, die sich auch im klassischen Batchbetrieb zeigen. Beispielsweise kann bei zunehmender Auftragsgröße oder einer größeren Anzahl an Aufträgen im System mit einem Anstieg der Entnahmedichte und somit einer Steigerung der erzielbaren Kommissionierleistung gerechnet werden. Analog zum Batchbetrieb können jedoch bei vielen großen Aufträgen auch Effekte auftreten, die sich negativ auf die Leistungsfähigkeit der dynamischen Auftragssteuerung auswirken können. Als Beispiel sei hier der Leistungsverlust des Sortiersystems durch einen Anstieg der rezirkulierenden Teile aufgeführt.

Durch die dynamische Arbeitsweise des Floating-Batch-Prinzips werden weitere Einflüsse relevant, die das Systemverhalten nachhaltig beeinflussen. Da die Auswahl eines Folgeauftrages erst unmittelbar bei dessen Anforderung erfolgt, bietet sich beispielsweise die Möglichkeit einer sehr kurzfristigen Festlegung der Auftragsreihenfolge. Dies kann sich in kürzeren Durchlaufzeiten, einer höheren Kapazitätsauslastung und einer besseren Termineinhaltung niederschlagen. 
Die Steigerung der Soll-Anzahl aktiver Aufträge lässt auf eine Erhöhung der Entnahmedichte im Kommissioniersystem und somit auf eine Leistungssteigerung schließen. Gleichzeitig steigt durch den zunehmenden Auftragsbestand im System jedoch auch die Auftragsdurchlaufzeit des einzelnen Auftrages.

Die Anzahl der Aufträge, die bei Erfüllung der Freigabekriterien (Freigabezeitpunkt) gleichzeitig in den aktuellen Auftragsstapel eingespielt werden, wird als Subbatchgröße bezeichnet. Unter bestimmten Umständen ist es sinnvoll, nicht jeden Auftrag unmittelbar durch einen neuen Auftrag zu ersetzen. Hierbei muss jedoch berücksichtigt werden, dass große Subbatches zu Verzögerungen bei der Freigabe neuer Aufträge im Kommissioniersystem führen, hierdurch die Entnahmedichte im Kommissioniersystem sinkt und dies letztlich zu einem Leistungsverlust führt.

Als wesentliche Einflussgrößen und Zielfaktoren der dynamischen Auftragssteuerung konnten im Rahmen des Projektes die in Abbildung 6 aufgezeigten Größen identifiziert werden.

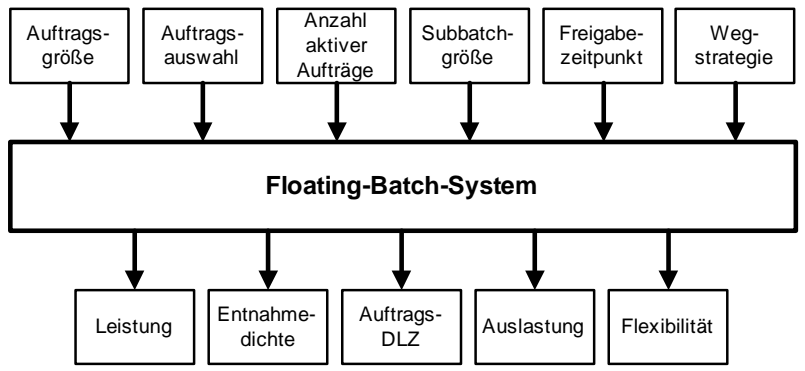

Abbildung 6: Einflussgrößen und Zielfaktoren der dynamischen Auftragssteuerung

Eine wichtige Einflussgröße auf die Effizienz und Leistungsfähigkeit eines Kommissioniersystems ist die Entnahmedichte. Bei der statischen Auftragssteuerung hängt diese von der Auftragsgröße und der Batchgröße ab. Prinzipiell gilt dieser Zusammenhang auch bei der dynamischen Auftragssteuerung, bei der jedoch die Anzahl der aktiven Aufträge die Batchgröße ersetzt. Darüber hinaus wird bei dieser Strategie die Entnahmedichte von weiteren Parametern beeinflusst. Dies sind zum einen die im System realisierte Wegstrategie und zum anderen die zeitliche Verzögerung zwischen der Entnahme des letzten Teils eines Auftrages und der Freigabe des Folgeauftrages.

Die Auswirkungen der Wegstrategien lassen sich durch die Betrachtung der Entnahmedichte innerhalb einer Kommissioniergasse verdeutlichen (Abb. 7). Der Bereich vor dem Kommissionierer weist eine hohe Entnahmedichte auf, da dieser Teil der Gasse noch nicht durchlaufen wurde. Im zurückliegenden Teil der Gasse wurden bereits alle Positionen kommissioniert. Durch das kontinuierliche Einspielen neuer Aufträge können hier bereits erste Anforderungen auftreten, dennoch wird die Entnahmedichte gering sein. Es kann davon ausgegangen werden, dass die Entnahmedichte umso höher sein wird, je länger der Kommissionierer einen bestimmten Teil des Systems nicht durchlaufen hat. Durchläuft der Kommissionierer, bedingt durch die vorgegebene Wegstrategie, einen Bereich des Kommissionierlagers bereits nach kurzer Zeit erneut (z.B. Umkehren am Gassenende), so ist für diesen Bereich mit einer im Vergleich zur mittleren Entnahmedichte geringeren Entnahmedichte zu rechnen. Vor diesem Hintergrund ist es für die dynamische Auftragssteuerung bei bestimmten Wegstrategien denkbar, dass die Entnahmedichte gegenüber einer statischen Auftragssteuerung abnimmt und es hierdurch zu einem Anstieg der Wegzeiten kommt, um dieselbe Entnahmemenge zu erzielen. Bei anderen Wegstrategien hingegen ist eine Erhöhung der Entnahmedichte und somit eine Reduzierung der Wegzeiten möglich.

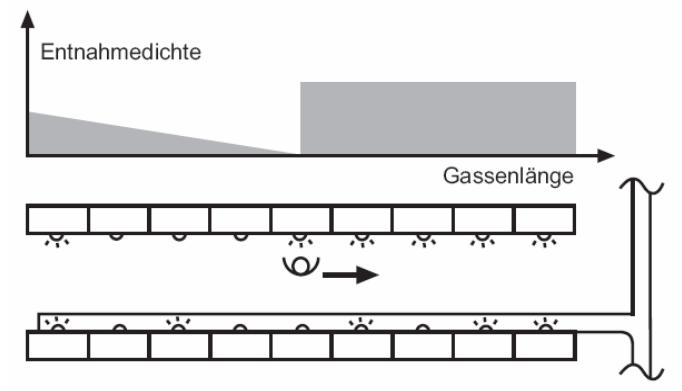

Abbildung 7: Entnahmedichte bei der dynamischen Auftragssteuerung

Auf Grund der Komplexität der Wirkzusammenhänge ist eine analytische Berechnung der Auswirkungen der verschiedenen Strategien zur Auftragssteuerung nicht mehr möglich. Daher wurde bei der Untersuchung des Systemverhaltens bei Integration einer dynamischen Auftragssteuerung die ereignisdiskrete Simulation als Hilfsmittel eingesetzt. 


\section{Modellierung und Versuchsdurchführung}

\subsection{Ableitung Modell}

Um im Rahmen des Forschungsprojektes verallgemeinerbare und praxisrelevante Ergebnisse zu erzielen, wurde eine umfassende Recherche und detaillierte Analyse realisierter zweistufiger Kommissioniersysteme durchgeführt. Da der Einsatz einer dynamischen Auftragssteuerung spezielle Anforderungen an die Systemstruktur stellt und somit nicht jedes zweistufige Kommissioniersystem für eine dynamische Auftragssteuerung geeignet ist, wurden mittels Besichtigungen, Fragebögen sowie durch Gespräche mit Planern und Betreibern grundsätzlich geeignete Systeme vorausgewählt. Für diese erfolgte eine Erfassung und Aufarbeitung aller für die weiteren Untersuchungen relevanten systembeschreibenden Kenngrößen und Parameter. Betrachtet wurden hierbei die Bereiche Kommissionierung, Sortierung sowie das Packsystem.

Aus den Erkenntnissen dieser Untersuchungen wurde ein typisches und somit repräsentatives Modellsystem entwickelt, das die Voraussetzungen für die anschließenden Untersuchungen am besten erfüllt und deshalb als Basis für die weiteren Arbeitsschritte herangezogen wurde.

Anhand dieses Modells wurde anschließend eine Vielzahl von Untersuchungen zu den Auswirkungen der dynamischen Auftragssteuerung auf das Systemverhalten zweistufiger Kommissioniersysteme durchgeführt. Im Folgenden werden kurz einige der gewonnenen Erkenntnisse dargestellt.

\subsection{Auswirkungen auf die Systemauslastung}

Bei den durchgeführten Versuchen bestätigte sich die Vermutung, dass bei der statischen Auftragssteuerung eine stark schwankende Systembelastung auftritt, während bei der dynamischen Auftragssteuerung eine deutlich gleichmäßigere Belastung zu beobachten ist (Abb. 8). Erwartungsgemäß sind die Kurvenverläufe bei Systemanlauf zunächst identisch. Ein Unterschied entsteht, sobald die ersten Endstellen vollständig entleert und Aufträge fertig gestellt sind.

Bei der statischen Auftragssteuerung wird ein neues Batch erst dann eingespielt, wenn alle Endstellen des Sortiersystems entleert wurden (FBoÜ) bzw. wenn alle Teile des Batches eingeschleust sind (FBmÜ). Hierdurch sinkt im Übergang zwischen zwei Batches die Auslastung des Kommissioniersystems und es resultieren Totzeiten. Bei der dynamischen Auftragssteuerung (DB) hingegen werden zu diesem Zeitpunkt neue Aufträge in den laufenden Auftragsstapel eingespielt, wodurch Totzeiten der Kommissionierer weitestgehend vermieden werden können und die Entnahmeleistung wieder ansteigt.

Durch die kontinuierliche Arbeitsweise des Floating-Batch-Prinzips kann daher im Gegensatz zum klassischen Batchbetrieb eine höhere und harmonischere Auslastung des Kommissionier- und Sortiersystems erreicht werden (Abb. 8).

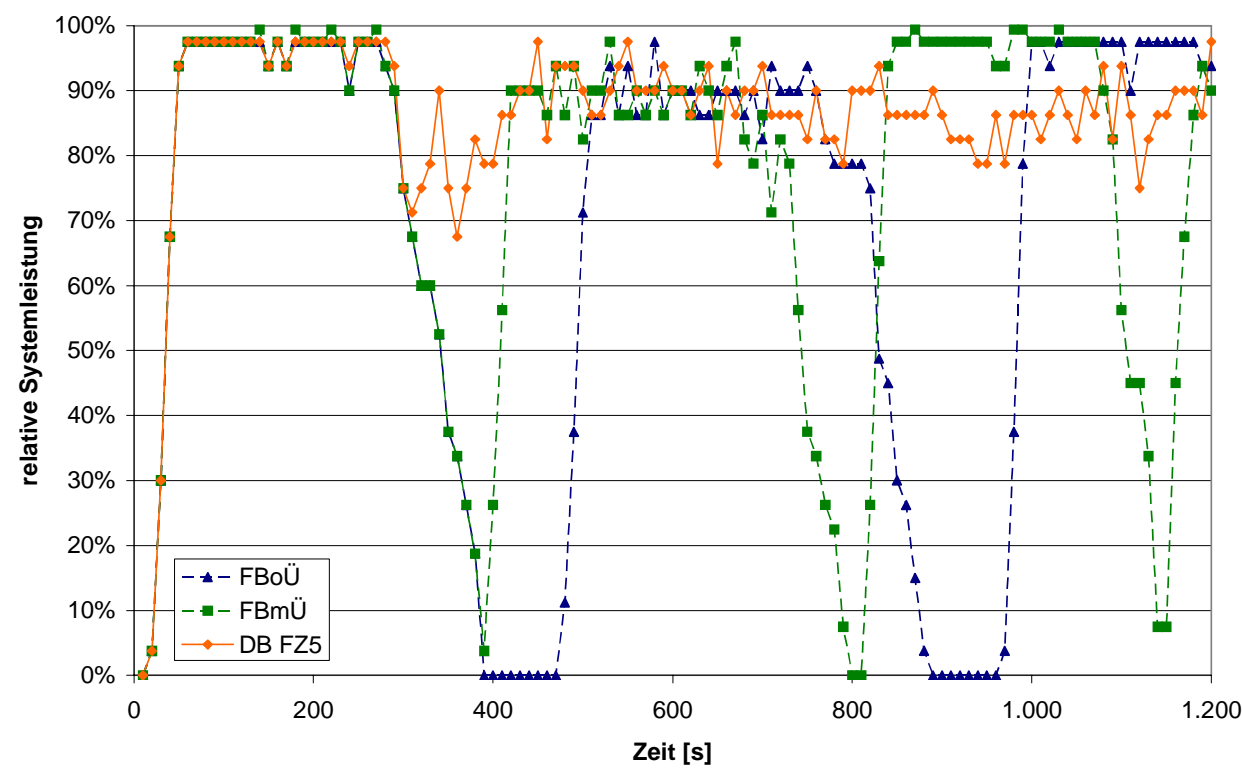

Abbildung 8: Relative Systemleistung in Abhängigkeit der Strategie zur Auftragssteuerung 


\subsection{Auswirkungen auf die Systemleistung}

Abbildung 9 zeigt die relative mittlere Systemleistung der alternativen Strategien zur Auftragssteuerung in Abhängigkeit der Auftragsgröße und des Freigabezeitpunktes. Hierbei zeigt sich wie erwartet, dass die mittlere Systemleistung der dynamischen Auftragssteuerung deutlich über der bei statischer Auftragssteuerung liegt. Dies ist wie bereits aufgezeigt darauf zurückzuführen, dass die im Batchbetrieb auftretenden Totzeiten weitestgehend vermieden werden können. Im Rahmen der Untersuchungen konnte bei einzelnen Wegstrategien zusätzlich eine Erhöhung der Entnahmedichte und somit eine Reduzierung der Wegzeiten erzielt werden. Bei allen Versuchen zeigt sich, dass die mittlere Leistung mit zunehmender Auftragsgröße ansteigt, da durch die größere Anzahl an Positionen auch die Entnahmedichte in der Kommissionierung ansteigt.

Die Freigabe neuer Aufträge bzw. eines neuen Batches ist bei Freigabezeitpunkt FZ5 an die vollständige Entleerung der Endstellen gekoppelt. Hierdurch entstehen im Falle einer statischen Auftragssteuerung hohe Totzeiten bei den Kommissionierern, da diese auf die Fertigstellung aller Aufträge im Packsystem warten müssen. Die Höhe der Totzeiten hängt in diesem Fall unmittelbar von der Leistung des Packsystems ab. Erst der langsamste Packer löst die Batchfreigabe aus.

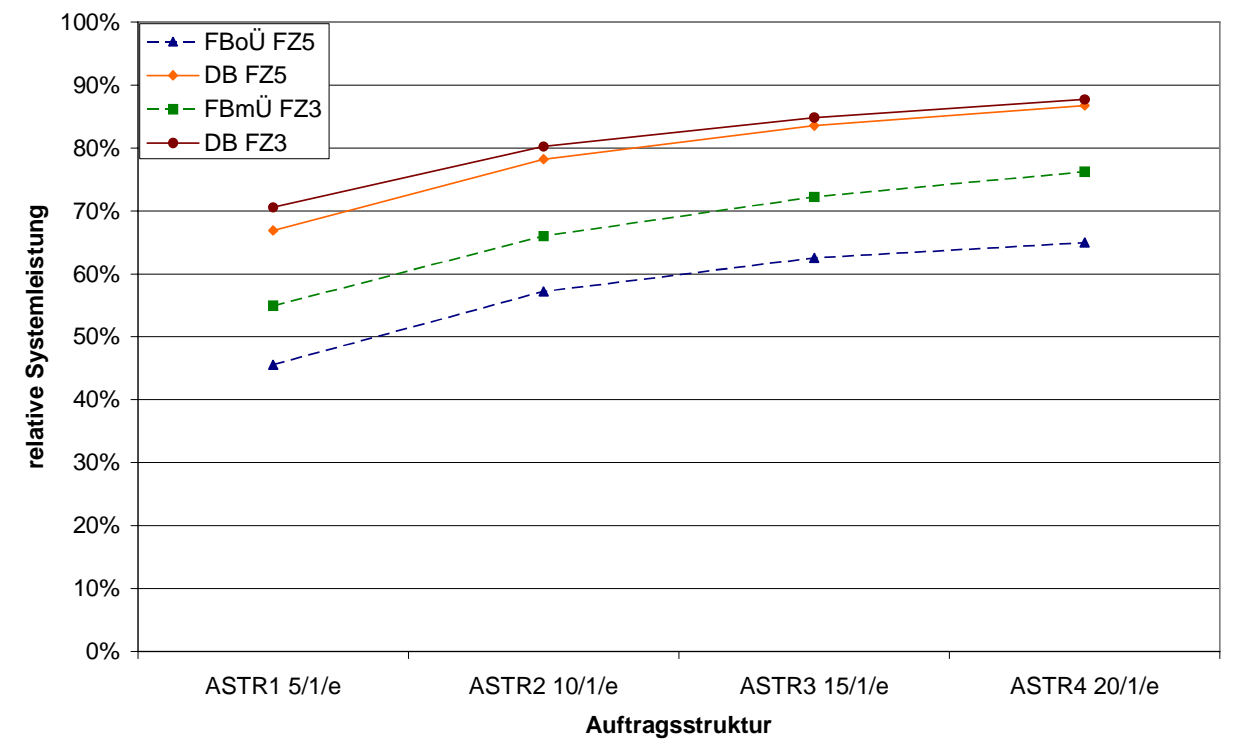

Abbildung 9: Relative Systemleistung in Abhängigkeit von Auftragsgröße und Freigabezeitpunkt

Bei Freigabezeitpunkt (FZ3) erfolgt die Freigabe neuer Aufträge bzw. eines neuen Batches bereits bei vollständiger Einschleusung. In diesem Fall ist die Leistung des Packsystems nicht mehr ausschlaggebend, so dass die Totzeiten deutlich reduziert werden können und die Leistung hierdurch gesteigert werden kann.

Während bei der statischen Auftragssteuerung der langsamste Packer ein neues Batch auslöst, stößt der schnellste Packer bei der dynamischen Auftragssteuerung die Freigabe eines neuen Auftrags an. Daher ist der Einfluss des Freigabezeitpunktes auf die Leistung bei der dynamischen Auftragssteuerung deutlich geringer.

\subsection{Auswirkungen auf die Flexibilität}

Eine wichtige Eigenschaft moderner Kommissioniersysteme ist die Möglichkeit flexibel auf kurzfristige Ereignisse reagieren zu können. Bei einer statischen Auftragssteuerung kann nur bedingt auf diese Ereignisse reagiert werden, da sie frühestens bei Beginn des folgenden Batches berücksichtigt werden können. Die Flexibilität eines Systems ist daher maßgeblich von der Laufzeit eines Batches abhängig. Im Extremfall sind weitere Batches bereits vorausberechnet, so dass sich die Möglichkeit zur Reaktion weiter einschränkt. Um diesem Mangel an Flexibilität beim klassischen Batchbetrieb zu begegnen, werden Eilaufträge in der Praxis teilweise als Sonderabläufe am System vorbei bearbeitet.

Bei der dynamischen Auftragssteuerung hingegen werden im eingeschwungenen Zustand kontinuierlich Aufträge fertig und können durch neue Aufträge ersetzt werden. Die Zeitdifferenz zwischen den Einspielzeitpunkten nacheinander eingespielter Aufträge wird als Subbatchtakt bezeichnet. Im Rahmen der durchgeführten Untersuchung lag der Subbatchtakt bei leistungsstarkem Kommissionier- und Sortiersystem bei unter einer Minute. Hierdurch kann mittels dynamischer Auftragssteuerung eine hohe Flexibilität gegenüber kurzfristigen Ereignissen gewährleistet werden. Kurzfristig eingegangene Kundenaufträge können beispielsweise über die Vergabe einer hohen Priorität somit sehr schnell in den normalen Ablauf integriert werden. 


\subsection{Auswirkungen auf die Durchlaufzeit}

Auswirkungen zeigt die Variation der Strategie zur Auftragssteuerung auch auf die Auftragsdurchlaufzeit. Abbildung 10 zeigt einen Vergleich der Durchlaufzeitverteilungen bei Variation der Strategie zur Auftragssteuerung.

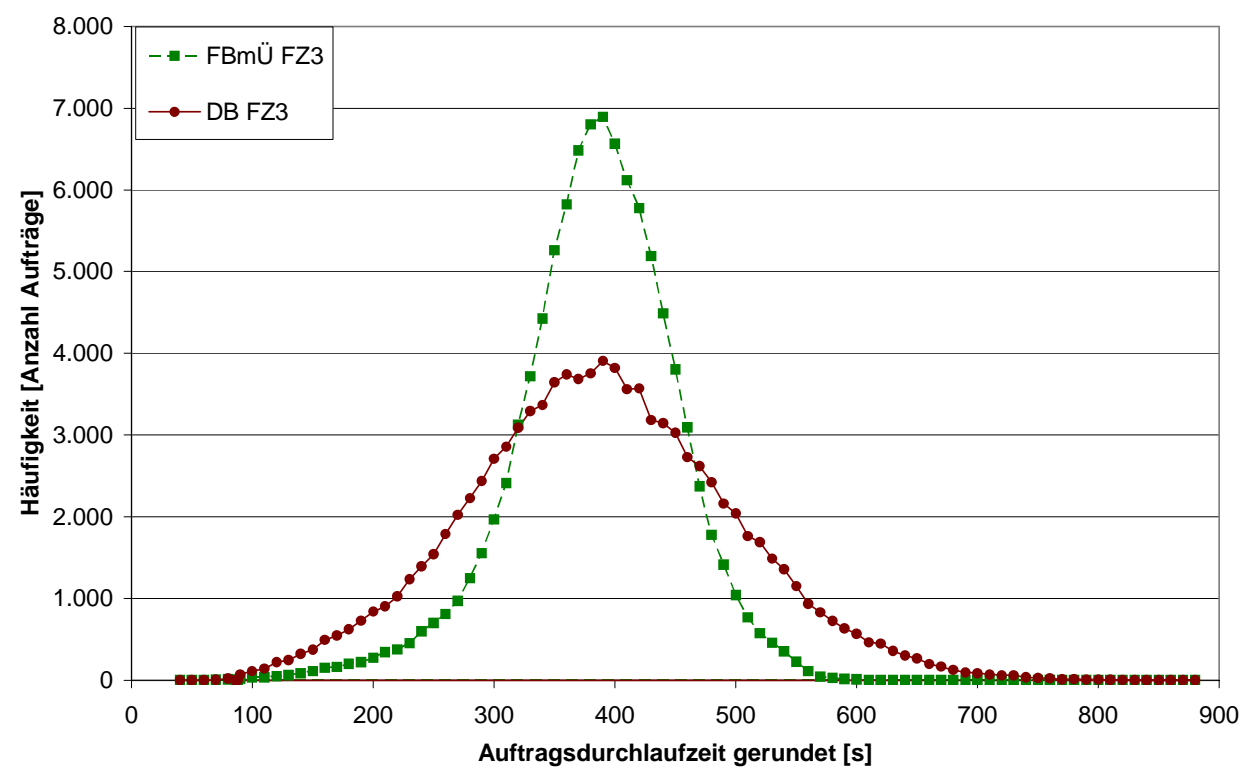

Abbildung 10: Einfluss der Strategie zur Auftragssteuerung auf die Auftragsdurchlaufzeit

Durch das Einspielen neuer Aufträge kommt es bei der dynamischen Auftragssteuerung zu einer Verzögerung von Positionen der bereits im Auftragsstapel vorhandenen Aufträge und somit zu einem Anstieg der Durchlaufzeit dieser Aufträge. Dies ist insbesondere bei Aufträgen mit vielen Positionen festzustellen. Andere Aufträge laufen schneller durch das System, was im Wesentlichen auf zwei Effekte zurückzuführen ist. Einerseits auf eine in bestimmten Fällen geringere Entnahmedichte gegenüber der Batchbearbeitung. Andererseits werden Aufträge eingespielt, deren Positionen schon nach einer sehr kurzen Wegstrecke vollständig kommissioniert werden. In diesen Fällen verkürzt sich die Durchlaufzeit gegenüber der statischen Auftragssteuerung.

Durch die stärkere Streuung der Auftragdurchlaufzeiten bei der dynamischen Auftragssteuerung wird die Vorhersage der Fertigstellungszeitpunkte für bestimmte Aufträge erschwert. Im realen Einsatz ist es erforderlich, Liefertouren so im Warenausgang bereitzustellen, dass beispielsweise die Abfahrtzeiten der jeweiligen LKWTouren berücksichtigt werden. Durch die stärkere Streuung der Durchlaufzeiten müssen daher gegebenenfalls größere Pufferflächen zur Verfügung stehen.

\section{Zusammenfassung}

Durch die durchgeführten Untersuchungen zu den Auswirkungen der dynamischen Auftragssteuerung auf das Systemverhalten zweistufiger Kommissioniersysteme wurden verallgemeinerbare und auf realisierte Kommissioniersysteme übertragbare Erkenntnisse abgeleitet. Es konnte eindeutig belegt werden, dass die dynamische Auftragssteuerung grundsätzlich für den Einsatz in zweistufigen Kommissioniersystemen geeignet ist. Auf Grund der dynamischen Arbeitsweise des Floating-Batch-Prinzips kann, im Vergleich zum klassischen Batchbetrieb, eine deutlich harmonischere und durchgehend hohe Auslastung der Teilsysteme erreicht werden und die Systemleistung signifikant gesteigert werden.

Mit Hilfe der gewonnenen Erkenntnisse lassen sich sowohl bei der Planung, als auch im Betrieb zweistufiger Kommissioniersysteme Rationalisierungspotenziale durch den Einsatz einer dynamischen Auftragssteuerung erschließen. In bereits bestehenden Systemen kann mit der existierenden Systemtechnik eine höhere Gesamtleistung erreicht werden, wodurch ggf. Investitionskosten für Systemerweiterungen eingespart werden können. Durch die erzielbare gleichmäßige hohe Auslastung lassen sich auch die Anforderungen an die erforderliche Leistung der Sortieranlage verringern und somit die erforderlichen Investitionskosten senken.

Weitere konkrete Optimierungspotenziale für die Praxis ergeben sich aus der Flexibilität gegenüber Eilaufträgen. So ist es im Gegensatz zum klassischen Batchbetrieb durch den Einsatz einer dynamischen Auftragssteuerung nach dem Floating-Batch-Prinzip möglich ein zweistufiges Kommissioniersystem auch dann wirtschaftlich zu betreiben, wenn eine kurzfristige Änderung der Auftragspriorisierung erforderlich ist. 


\section{Literatur}

[Bozer1983]

[Bozer1985]

[Bozer1988]

[Gudehus2005]

[Johnson1998]

[Jünemann1999]

[Meller1997]

[Radtke2000]
Bozer, Yavuz A.; Sharp, Gunter P.; Throughput analysis of order accumulation and sortation systems; MHRC report TR-83-07, Atlanta, 1983

Bozer, Yavuz A.; Sharp, Gunter P.; An empirical evaluation of a general purpose automated order accumulation and sortation system used in batch picking; IN: Material Flow (1985) 2, S. 111-131

Bozer, Yavuz A.; Quiroz, Marco A.; Sharp, Gunter P.; An evaluation of alternative control strategies for automated order accumulation and sortation systems; IN Material Flow (1988) 4, S. 265-282

Gudehus, Timm; Logistik - Grundlagen, Strategien, Anwendungen; Berlin (usw.): Springer, 2005

Johnson, M. Eric; The impact of sorting strategies on automated sortation system performance; IN: IIE Transactions (1998) 30, S. 67-77

Jünemann, Reinhardt; Schmidt, Thorsten; Materialflusssysteme - Systemtechnische Grundlagen; Berlin (usw.): Springer, 1999

Meller, Russel D.; Optimal order-to-lane assignment in an order accumulation / sortation system; IN: IIE Transactions, (1997) 29, S. 293-301

Radtke, A.: Beitrag zur Entwicklung optimierter Betriebsstrategien für Sortiersysteme. Dissertation, Universität Dortmund, 2000 